Journal of Mathematics and Statistics 3 (1): 17-20, 2007

ISSN 1549-3644

(C) 2007 Science Publications

\title{
Global Behavior of a Higher Order Difference Equation
}

\author{
${ }^{1}$ Alaa.E. Hamza and ${ }^{2}$ R. Khalaf-Allah \\ ${ }^{1}$ Department of Mathematics, Faculty of Science, Cairo University, Giza, 12211, Egypt \\ ${ }^{2}$ Department of Mathematics, Faculty of Science, Helwan University, Cairo, 11795, Egypt
}

Abstract: In this work we study the asymptotic stability of the nonnegative equilibrium points of the
difference equation $x_{n+1}=\frac{A x_{n-1}}{B+C \prod_{i=l}^{k} x_{n-2 i}}, n=0,1, \ldots$ where $A, B, C$ are nonnegative real numbers and $l, k$ are nonnegative integers, $l \leq k$. We discuss the conditions under which there exist prime period two solutions and semicycles. Finally we investigate the oscillation and the existence of unbounded solutions.

Key words: Difference equation, periodic solution, globally asymptotically stable

\section{INTRODUCTION}

Difference equations have always played an important role in the construction and analysis of mathematical models of biology, ecology, physics, economic processes, etc ${ }^{[1]}$.

The study of nonlinear rational difference equations of higher order is of paramount importance, since we still know so little about such equations. Cinar $^{[2]}$ examined the global asymptotic stability of all positive solutions of the rational difference equation

$$
x_{n+1}=\frac{a x_{n-1}}{1+b x_{n} x_{n-1}}, n=0,1, . .
$$

Where $a$ and $b$ are nonnegative real numbers.

Xiaofan yang et al. ${ }^{[3]}$ investigated the asymptotic behavior of solutions of the difference equations

$x_{n+1}=\frac{a x_{n}+b x_{n-1}}{c+d x_{n} x_{n-1}}, n=0,1, \ldots$

Where $a \geq 0$ and $b, c, d$ are nonnegative real numbers.

Gibbons et al. $^{[4]}$ investigated the global asymptotic behavior of the difference equation

$x_{n+1}=\frac{\alpha+\beta x_{n-1}}{\gamma+x_{n}}, n=0,1, \ldots$

where $\beta>0$ and $\alpha, \gamma \geq 0$.

In this study, we study the global asymptotic stability of the difference equation

$$
x_{n+1}=\frac{A x_{n-1}}{B+C \prod_{i=l}^{k} x_{n-2 i}}, n=0,1, \ldots
$$

where $A, B, C$ are nonnegative real numbers and $l, k$ are nonnegative integers, $l \leq k$.

The following particular cases can be obtained:
1. When $A=0$, equation (1.2) reduces to the equation $x_{n+1}=0, n=0,1, \ldots$

2. When $B=0$, equation (1.2) reduces to the equation $\quad x_{n+1}=\frac{A x_{n-1}}{C \prod_{i=l}^{k} x_{n-2 i}}, n=0,1, \ldots, \quad$ this equation can be reduced to the linear difference equation

$y_{n+1}-y_{n-1}+y_{n-2 l}+\cdots+y_{n-2 k}=\gamma, n=0,1, \ldots$

by taking $y_{n}=x_{n}, \gamma=\ln \frac{A}{C}$

3. When $C=0$, equation (1.2) reduces to the equation $x_{n+1}=\frac{A}{B} x_{n-1}, n=0,1, \ldots$ which is a linear difference equation.

4. When $l=k=0$, and $C=1$ equation (1.2) yields equation (1.1) with $\alpha=0$. For various values of $l$ and $k$, we can get more equations.

Preliminaries: Consider the difference equation

$x_{n+1}=f\left(x_{n}, x_{n-1}, \ldots, x_{n-k}\right), n=0,1, \ldots$

Where $f: R^{k+1} \rightarrow R$

Definition 2.1 ${ }^{[5]}$ : An equilibrium point for equation (2.1) is a point $\bar{x} \in R$ such that $\bar{x}=f(\bar{x}, \bar{x}, \ldots \bar{x})$.

\section{Definition $2.2^{[5]}$}

1. An equilibrium point $\bar{x}$ for equation (2.1) is called locally stable if for every $\varepsilon>0, \exists \delta>0$ such that every solution $\left\{x_{n}\right\}$ with initial conditions $\left.x_{-k}, x_{-k+1}, \ldots, x_{0} \in\right] \bar{x}-\delta, \bar{x}-\delta[\quad$ is such

Corresponding Author: $\quad$ R Khalaf-Allah, Department of Mathematics, Faculty of Science, Helwan University, Cairo, 11795, Egypt 
that $\left.x_{n} \in\right] \bar{x}-\varepsilon, \bar{x}+\varepsilon[\forall n \in N$. Otherwise $\bar{x}$ is said to be unstable.

2. The equilibrium point $\bar{x}$ of equation (2.1) is called locally asymptotically stable if it is locally stable and there exists $\gamma>0$ such that for any initial conditions $\left.\quad x_{-k}, x_{-k+1}, \ldots, x_{0} \in\right] \bar{x}-\gamma, \bar{x}-\gamma[, \quad$ the corresponding solution $\left\{x_{n}\right\}$ tends to $\bar{x}$.

3. An equilibrium point $\bar{x}$ for equation (2.1) is called global attractor if every solution $\left\{x_{n}\right\}$ converges to $\bar{x}$ as $n \rightarrow \infty$.

4. The equilibrium point $\bar{x}$ for equation (2.1) is called globally asymptotically stable if it is locally asymptotically stable and global attractor. (2.1) is

The linearized equation associated with equation

$$
y_{n+1}=\sum_{i=0}^{k} \frac{\partial f}{\partial x_{n-i}}(\bar{x}, \bar{x}, \ldots, \bar{x}) y_{n-i}, n=0,1, \ldots
$$

the characteristic equation associated with equation (2.2) is

$$
\lambda^{k+1}-\sum_{i=0}^{k} \frac{\partial f}{\partial x_{n-i}}(\bar{x}, \bar{x}, \ldots, \bar{x}) \lambda^{k-i}=0
$$

Theorem 2.3 ${ }^{[5]}$ : Assume that $f$ is a $C^{1}$ function and let $\bar{x}$ be an equilibrium point of equation (2.2). Then the following statements are true:

1. If all roots of equation (2.3) lie in the open disk $|\lambda|<1$, then $\bar{x}$ is locally asymptotically stable.

2. If at least one root of equation (2.3) has absolute value greater than one, then $\bar{x}$ is unstable.

The change of variables $x_{n}=\sqrt[k-l+1]{\frac{B}{C}} y_{n}$ reduces equation (1.2) to the difference equation

$$
y_{n+1}=\frac{\gamma y_{n-1}}{1+\prod_{i=1}^{k} y_{n-2 i}}, n=0,1, \ldots
$$

where $\gamma=\frac{A}{B}$.

Linearized stability analysis: In this section we study the asymptotic stability of the nonnegative equilibrium points of equation (2.4). We can see that equation (2.4) has two nonnegative equilibrium points $\bar{y}=0$ and $\bar{y}=\sqrt[k-l+1]{\gamma-1}$ when $\gamma>1$ and the zero equilibrium only when $\gamma \leq 1$.

The linearized equation associated with equation (2.4) about $\bar{y}$ is

$$
\begin{aligned}
& z_{n+1}-\frac{\gamma}{1+\bar{y}^{k-l+1}} z_{n-1}+\frac{\gamma \bar{y} \bar{y}^{k-l}}{\left(1+\bar{y}^{k-l+1}\right)^{2}} \sum_{i=l}^{k} z_{n-2 i} \\
& =0, n=0,1, \ldots
\end{aligned}
$$

the characteristic equation associated with this equation is

$$
\begin{aligned}
& \lambda^{2 k+1}-\frac{\gamma}{1+\bar{y}^{k-l+1}} \lambda^{2 k-1}+ \\
& \frac{\gamma y \bar{y}^{k-l}}{\left(1+\bar{y}^{k-l+1}\right)^{2}} \sum_{i=l}^{k} \lambda^{2 k-2 i}=0 .
\end{aligned}
$$

We summarize the results of this section in the following theorem.

\section{Theorem 3.1}

1. If $\gamma<1$, then the zero equilibrium point is locally asymptotically stable.

2. If $\gamma>1$, then the equilibrium points $\bar{y}=0$ and $\bar{y}=\sqrt[k-l+1]{\gamma-1}$ are unstable (saddle points).

Proof: The linearized equation associated with equation (2.4) about $\bar{y}=0$ is

$z_{n+1}-\gamma z_{n-1}=0, n=0,1, \ldots$

the characteristic equation associated with this equation is

$\lambda^{2 k+1}-\gamma \lambda^{2 k-1}=0$.

So $\lambda=0, \pm \sqrt{\gamma}$.

1. If $\gamma<1$, then $|\lambda|<1$ for all roots and $\bar{y}=0$ is locally asymptotically stable.

2. If $\gamma>1$, it follows that $\bar{y}=0$ is unstable (saddle point).

The linearized equation (3.1) about $\bar{y}=\sqrt[k-l+1]{\gamma-1}$ becomes

$z_{n+1}-z_{n-1}+\left(1-\frac{1}{\gamma}\right) \sum_{i=l}^{k} z_{n-2 i}=0, n=0,1, \ldots$

the associated characteristic equation is

$\lambda^{2 k+1}-\lambda^{2 k-1}+\left(1-\frac{1}{\gamma}\right) \sum_{i=l}^{k} \lambda^{2 k-2 i}=0$.

Let $f(\lambda)=\lambda^{2 k+1}-\lambda^{2 k-1}+\left(1-\frac{1}{\gamma}\right) \sum_{i=l}^{k} \lambda^{2 k-2 i}$.

We can see that $f(\lambda)$ has a root in $(-\infty,-1)$. Then the point $\bar{y}=\sqrt[k-l+1]{\gamma-1}$ is unstable (saddle point).

Global behavior of equation (2.4)

Theorem 4.1: If $\gamma<1$, then the zero equilibrium point is globally asymptotically stable.

Proof: Let $\left\{y_{n}\right\}$ be a solution of equation (2.4). Hence $y_{n+1}=\frac{\gamma y_{n-1}}{1+\prod_{i=l}^{k} y_{n-2 i}}<\gamma y_{n-1}, n=0,1, \ldots$. This implies that $\lim _{n \rightarrow \infty} y_{n}=0$. 
In view of theorem (3.1), $\bar{y}=0$ is globally asymptotically stable.

Existence of prime period two solutions: This section is devoted to discuss the condition under which there exist prime period two solutions.

Theorem 5.1: A necessary and sufficient condition for equation (2.4) to have a prime period two solution is that $\gamma=1$. In this case the prime period two solution is of the form $\ldots, 0, \varphi, 0, \varphi, 0, \ldots$ where $\varphi>0$. Furthermore, every solution converges to a period two solution.

Proof

Sufficiency: Let $\gamma=1$, then for every $\varphi>0$, we have $\ldots, 0, \varphi, 0, \varphi, 0, \ldots$ is a prime period two solution.

Necessity: Assume that equation (2.4) has a prime period two solution $\ldots, \psi, \varphi, \psi, \varphi, \psi, \ldots$ then $\varphi=\frac{\gamma \varphi}{1+\psi^{k-l+1}}, \psi=\frac{\gamma \psi}{1+\varphi^{k-l+1}}$.

Hence

$(\varphi-\psi)+\varphi \psi\left(\psi^{k-l}-\varphi^{k-l}\right)=\gamma(\varphi-\psi)$,

this implies

$\varphi \psi\left(\frac{\psi^{k-l}-\varphi^{k-l}}{\psi-\varphi}\right)=1-\gamma$.

So $\gamma \leq 1$. Similarly,

$$
\varphi \psi \frac{\left(\psi^{k-l}+\varphi^{k-l}\right)}{(\varphi+\psi)}=\gamma-1 \text {. }
$$

So $\gamma \geq 1$. Therefore $\gamma=1$. From (5.2) we have $\varphi \psi=0$ and the solution is of the form $\ldots, 0, \varphi, 0, \varphi, 0, \ldots$ with $\varphi>0$.

Now let $\left\{y_{n}\right\}_{n=-2 k}^{\infty}$ be a solution of equation (2.4) with $\gamma=1$.

Then

$$
y_{n+1}=\frac{y_{n-1}}{1+\prod_{i=l}^{k} y_{n-2 i}}<y_{n-1}, n=0,1, \ldots
$$

and so the even terms $\left\{y_{2 n}\right\}_{n=-k}^{\infty}$ decreases to a limit $\varphi$ and the odd terms $\left\{y_{2 n+1}\right\}_{n=-k}^{\infty}$ decreases to a limit $\psi$, where $\varphi=\frac{\varphi}{1+\psi^{k-l+1}}, \psi=\frac{\psi}{1+\varphi^{k-l+1}}$.

Then $\psi^{k-l+1} \varphi=0$ and $\varphi^{k-l+1} \psi=0$. Therefore, $\left\{y_{n}\right\}_{n=-2 k}^{\infty}$ converges to the periodic solution $\ldots, 0, \varphi, 0, \varphi, 0, \ldots$ with $\varphi>0$.

Semicycle analysis: Here we discuss the existence of semicycles. We need the following theorem to obtain the main result of this section.
Theorem 6.1: Assume that $f \in C\left(\left[0, \infty\left[{ }^{2 k+1},[0, \infty[)\right.\right.\right.$ is increasing in the even arguments and decreasing in the others. Let $\bar{y}$ be an equilibrium point for the difference equation

$y_{n+1}=f\left(y_{n}, y_{n-1}, \ldots, y_{n-2 k}\right), n=0,1, \ldots$

Let $\left\{y_{n}\right\}_{n=-2 k}^{\infty}$ be a solution of equation (6.1) such that either, $\quad\left(C_{1}\right) \quad y_{-2 k}, y_{-2 k+2}, \ldots, y_{0}>\bar{y}, \quad$ and $y_{-2 k+1}, y_{-2 k+3}, \ldots, y_{-1}<\bar{y}$.

Or

$\left(C_{2}\right) \quad y_{-2 k}, y_{-2 k+2}, \ldots, y_{0}<\bar{y}$ and $y_{-2 k+1}, y_{-2 k+3}, \ldots, y_{-1}>\bar{y}$. is satisfied, then $\left\{y_{n}\right\}_{n=-2 k}^{\infty}$ oscillates about $\bar{y}$ with semicycles of length one.

Proof: Assume that $f$ is increasing in the even arguments and decreasing in the others.

Let $f$ be satisfying condition $\left(C_{1}\right)$ we have $y_{1}=f\left(y_{0}, y_{-1}, \ldots, y_{-2 k}\right)<f\left(\bar{y}, y_{-1}, \bar{y}, \ldots, \bar{y}\right)<$ $f(\bar{y}, \bar{y}, \bar{y}, \ldots, \bar{y})=\bar{y}$,

$y_{2}=f\left(y_{1}, y_{0}, \ldots, y_{-2 k+1}\right)>f\left(\bar{y}, y_{0}, \bar{y}, \ldots, \bar{y}\right)>$ $f(\bar{y}, \bar{y}, \bar{y}, \ldots, \bar{y})=\bar{y}$.

By induction we obtain the result.

If $f$ satisfies condition $\left(C_{2}\right)$, we can prove the result similarly.

Corollary 6.2: Assume that $\gamma>1$ and let $\left\{y_{n}\right\}_{n=-2 k}^{\infty}$ be a solution of equation (2.4) such that either $\left(C_{1}\right)$ or $\left(C_{2}\right)$ is satisfied. Then $\left\{y_{n}\right\}_{n=-2 k}^{\infty}$ oscillates about the positive equilibrium point $\bar{y}=\sqrt[k-l+1]{\gamma-1}$ with semicycles of length one.

Proof: The proof follows directly from the previous theorem.

Existence of unbounded solutions: Finally we show that, under certain initial conditions, unbounded solution will be obtained.

Theorem 7.1: Assume that $\gamma>1$. Let $\left\{y_{n}\right\}_{n=-2 k}^{\infty}$ be a solution of equation (2.4) and $\bar{y}=\sqrt[k-l+1]{\gamma-1}$, the positive equilibrium point. Then the following statements are true:

1. If $y_{-2 k}, y_{-2 k+2}, \ldots, y_{0}>\bar{y} \quad$ and $y_{-2 k+1}, y_{-2 k+3}, \ldots, y_{-1}<\bar{y}, \quad$ then $\left\{y_{2 n}\right\}_{n=-k}^{\infty}$ increases to $\infty$ and $\left\{y_{2 n+1}\right\}_{n=-k}^{\infty}$ decreases to 0 . 
2. If $y_{-2 k}, y_{-2 k+2}, \ldots, y_{0}<\bar{y}$ and $y_{-2 k+1}, y_{-2 k+3}, \ldots, y_{-1}>\bar{y}$, then $\left\{y_{2 n}\right\}_{n=-k}^{\infty}$ decreases to 0 and $\left\{y_{2 n+1}\right\}_{n=-k}^{\infty}$ increases to $\infty$.

\section{Proof}

1. Let $\left\{y_{n}\right\}_{n=-2 k}^{\infty}$ be a solution of equation (2.4) with initial conditions, $y_{-2 k}, y_{-2 k+2}, \ldots, y_{0}>\bar{y}$ and $y_{-2 k+1}, y_{-2 k+3}, \ldots, y_{-1}<\bar{y}$. Then

$$
y_{2 n+2}=\frac{\gamma y_{2 n}}{1+\prod_{i=l}^{k} y_{2 n-2 i+1}}>\frac{\gamma y_{2 n}}{1+\bar{y}^{k-l+1}}=y_{2 n}
$$

and

$$
y_{2 n+3}=\frac{\gamma y_{2 n+1}}{1+\prod_{i=1}^{k} y_{2 n-2 i+2}}<\frac{\gamma y_{2 n+1}}{1+\bar{y}^{k-l+1}}=y_{2 n+1} .
$$

So $\left\{y_{2 n}\right\}_{n=-k}^{\infty}$ increases to $\infty$ and $\left\{y_{2 n+1}\right\}_{n=-k}^{\infty}$ decreases to 0 .

2. The proof is similar and will be omitted.

\section{ACKNOWLEDGMENT}

Many thanks to Dr. Adel El-Tohamy for his help and support.

\section{REFERENCES}

1. Mickens, R.E., 1990. Difference Equations, Theory and Applications. 2nd Edn, Van Nostrand Renhold.

2. Cinar, C., 2004. On the positive solution of the difference equation $x_{n+1}=\frac{a x_{n-1}}{1+b x_{n} x_{n-1}}$. Appl. Math. Comput., 156: 587-590.

3. Yang, X., W. Su, B. Chen, G. M. Megson and D. J. Evans, 2004. On the recursive sequence $x_{n+1}=\frac{a x_{n}+b x_{n-1}}{c+d x_{n} x_{n-1}} \quad$. Appl. Mathe. Comput., doi:10.1016/j.amc.2004.03.023.

4. Gibbons, C.H., M.R.S. Kulenović and G. Ladas, 2000. On the recursive sequence $x_{n+1}=\frac{\alpha+\beta x_{n-1}}{\gamma+x_{n}}$. Math. Sci. Res. Hot-Line, 4: 1-11.

5. Kocic, V.L. and G. Ladas, 1993. Global Behavior of Nonlinear Difference Equations of Higher Order with Applications. Kluwer Academic, Dordrecht. 